\title{
Background Removal and Data Analysis for Low-Loss Transmission Electron Energy-Loss Spectroscopy
}

\author{
Bryan W. Reed and Mehmet Sarikaya
}

Mater. Sci. \& Eng., Box 352120, University of Washington, Seattle, WA 98195, USA

In recent years, electron energy-loss spectroscopy (EELS) in the transmission electron microscope (TEM) has begun to receive its due recognition as a powerful and highly quantitative measurement technique. The tools for advanced numerical analysis of inner-shell edge spectra are well developed, widely disseminated, and continually improved upon by a large community of researchers. In comparison, data analysis in the low-loss regime (typically defined as energy losses below $\sim 50 \mathrm{eV}$ ) has received relatively little attention. Low-loss data analysis is almost invariably limited to Fourier deconvolution,[1] Kramers-Kronig analysis,[1] and sometimes curve fitting for parameter extraction.[2] Detailed error analyses are almost unheard of.

Our research involving aloof EELS of carbon nanotubes[3] demanded the maximum possible extraction of meaningful information from the spectra, including artifactfree removal of the zeroloss background, accurate tracking of peak heights, widths and positions throughout the data sets, and precise quantification of uncertainties. We have developed a complete set of tools to accomplish all of these goals, [4] and are making the software generally available via the world wide web.[5] The software has the following features:

- Removes zero-loss background from low-loss spectra with the minimum possible generation of artifacts, down to $\sim 2 \mathrm{eV}$ with current TEM/EELS systems. The unique "splinefit" algorithm we used should enable near-future monochromated TEM/EELS systems to extract meaningful numbers well into the infrared.

- Uses $\chi^{2}$ techniques and a well-tested semi-empirical model of the random error in the data produced by a spectrometer to allow the user to make informed judgments of the meaningfulness of features in the extracted data.

- Incorporates curve fits to multiple Lorentzian peaks (a functional form that we have shown to be particularly well suited to aloof EELS of nanoparticles) for extraction of excitation parameters, with uncertainties.

- Sufficiently intelligent parameter-guessing and optimization routines to run autonomously once it has been tuned on a particular data set.

- Runs in Mathematica, for portability and ease of maintenance. Able to export data for further processing by other software.

Compared with the Fourier techniques in common use for low-loss background removal, our algorithm is less sensitive to arbitrary parameters, produces fewer artifacts, extracts information to lower energies, and is more consistent with further data analysis including advanced statistical techniques. We will discuss these advantages, and describe the method as illustrated in Figs 1-3. The algorithm can consistently extract not only the $5 \mathrm{eV} \pi$ plasmon peak but also the $2-3 \mathrm{eV} \pi$ to $\pi^{*}$ interband transition peak (Fig. 4) in the low-loss EELS obtained from single carbon nanotubes and nanotube bundles. [3,4]. 
[1] R. F. Egerton, Electron Energy Loss Spectroscopy in the Electron Microscope, Second Edition (Plenum, New York, 1996).

[2] H. Cohen et al, Phys. Rev. Lett. 80 (1998) 782.

[3] B. W. Reed and M. Sarikaya, Phys. Rev. B 64 (2001) 195404.

[4] B. W. Reed and M. Sarikaya, Ultramicroscopy (2002) (to be published).

[5] hittp:///depts. washington edu/bionano/EELLS

[6] Supported by NSF Grant DMR $99 \overline{7} 8 \overline{3} \overline{5}$.
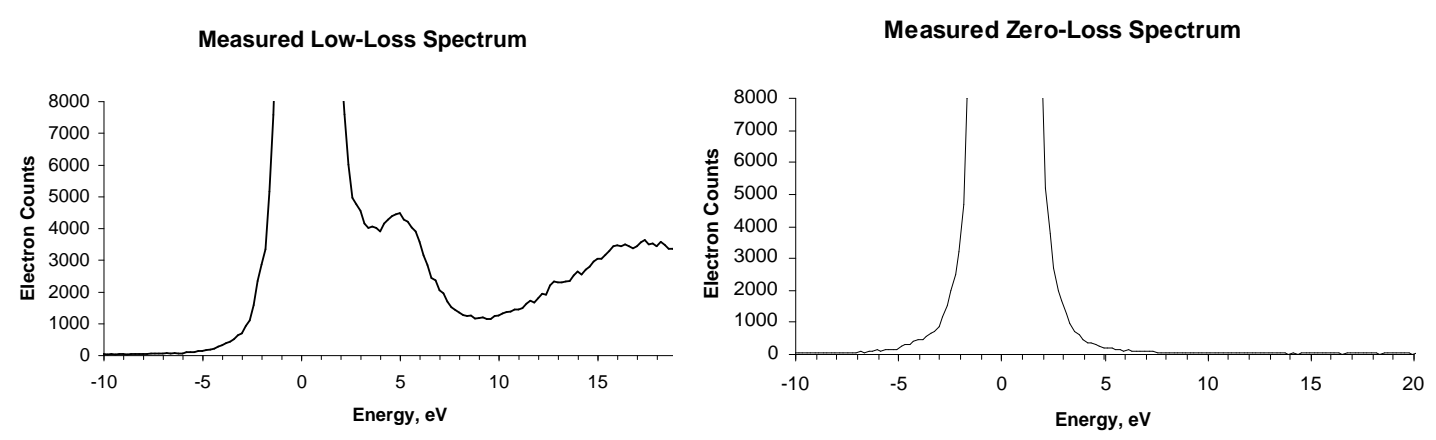

FIG. 1. Measured low-loss and background spectra for a carbon nanotube bundle.

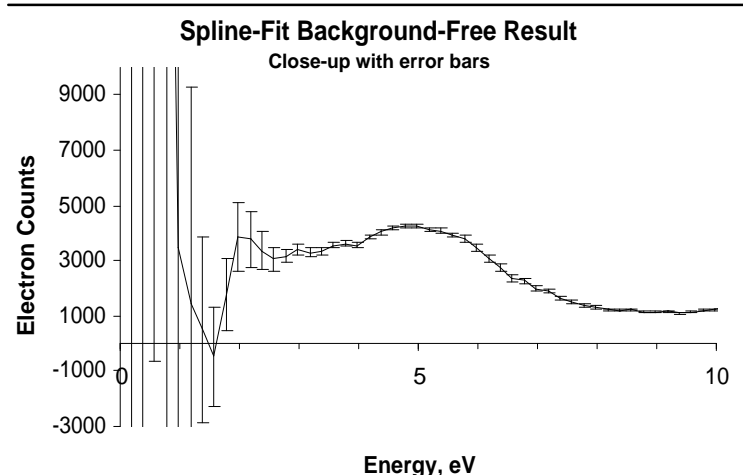

FIG. 2. Results of the background subtraction, with error bars.[4]

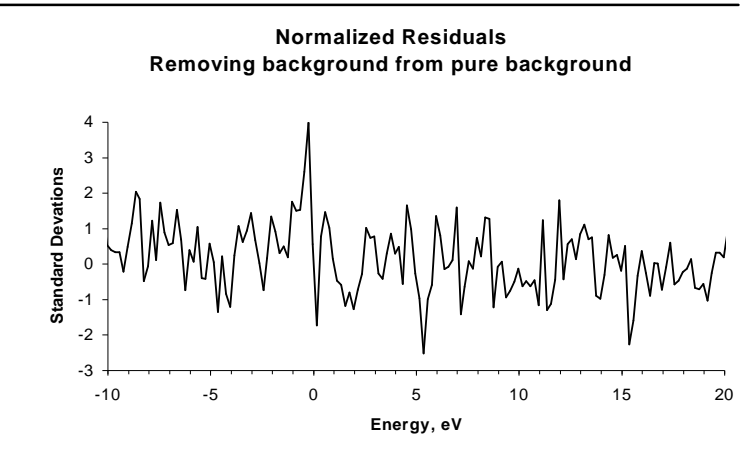

FIG. 3. Demonstration of correctness of error estimate and lack of artifacts. Should look like random noise.[4] (a)

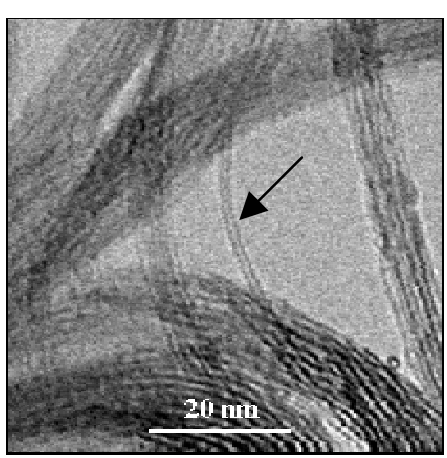

(b)

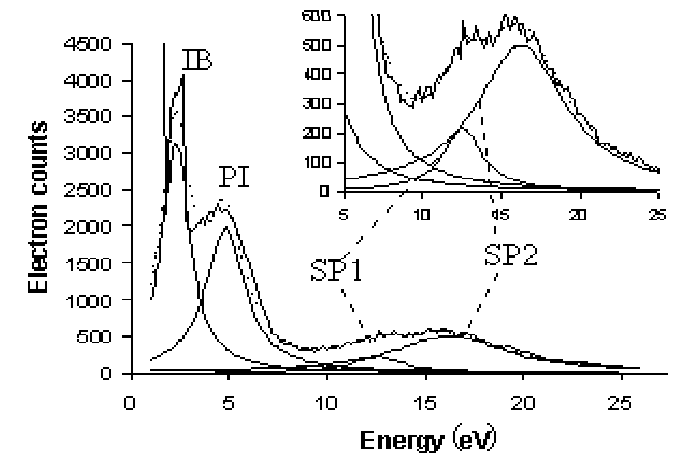

Fig. 4 (a) STEM image of carbon nanotube bundles, and a single nanotube (arrow). (b) Lorentzian decomposition of an aloof EELS spectrum of a nanotube, showing $\pi-\pi *$ interband transition (IB), $\pi$ plasmon (PI) and two surface plasmons (SP) in different polarizations. Inset is a closeup of the same data.[3] 